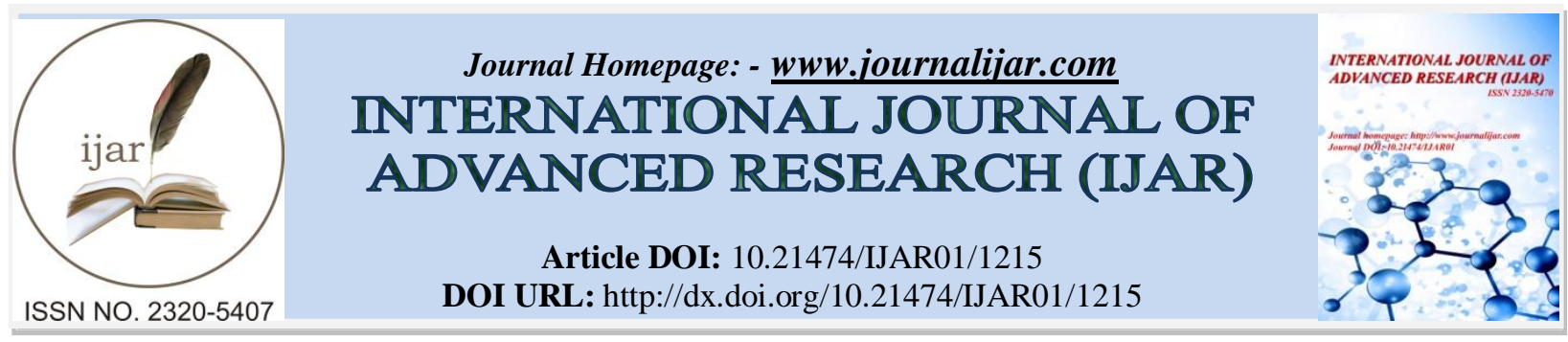

RESEARCH ARTICLE

\title{
MATUMIZI YA VISAIDIZI VYA UFUNDISHAJI MSAMIATI WA KISWAHILI KATIKA SHULE ZA MSINGI ZA UMMA, KAUNTI YA KISUMU MASHARIKI, KENYA.
}

Kamau Hellen Wambui, Pro. Samson Ondigi, Dr. Hamisi Babusa.

Kenyatta University, School of Education, P. O. Box 43844, Nairobi, Kenya.

\section{Manuscript Info}

Manuscript History

Received: 19 June 2016

Final Accepted: 14 July 2016

Published: August 2016

Key words: -

Dhana, Ensiklopidia, Expository, Heuristic, Kiunzi, Nyenzo, Mbinu, Mfawidhi, Mwelekeo-fikira, Pendekezo, Teknoelimu, Uhalali, Uaminifu, Kutathmini

\begin{abstract}
Utafiti huu ulishughulikia uchunguzi wa matumizi ya vifaa vya ufundishaji wa msamiati wa kiswahili katika shule za msingi za umma, Kaunti Ndogo ya Kisumu Mashariki, Kenya. Malengo aliyozingatia mtafiti katika utafiti huu yalikuwa pamoja na kutambua changamoto zilizopo wakati wa kutumia vinyago, video na chati katika somo la msamiati, kudadisi sera zilizopo za ukaguzi ili kudhibiti utumizi wa video, chati na vinyago ili kusaidia uelewekaji wa msamiati na kuchunguza udhaifu na ufaafu uliopo katika ufundishaji msamiati kwa kutumia video, chati na vinyago katika somo la Kiswahili. Utafiti ulilenga idadi ya walimu kumi wa Kiswahili katika Shule za msingi katika Kaunti Ndogo ya Kisumu Mashariki. Baadhi ya hizi mtafiti aliteuwa kwa bahati nasibu walengwa walioteuliwa kama hivi walimu wakuu kumi, walimu wa Kiswahili kumi na wanafunzi kumi. Walengwa wote walikuwa mia nane ishirini ambao waliimbua asilimia kumi, basi idadi ilio hojiwa ikawa themanini na wawili. Hojaji zilikuwa tatu za walimu wakuu, walimu wa Kiswahili. Utafiti ulichanganuliwa kwa kutumia SPSS kuonyesha idadi katika alama za asilimia na uradidi. Baada ya kutafiti na kuchanganua data, mtafiti alipendekeza haja ya walimu wa Kiswahili kuwa na vikao katika majopo. Mtafiti pia aona kuwa kuna haja washika dau wote wa elimu kufufua taasisi za Kaunti ili kukuza hitaji la uundaji visaidizi ili walimu wapate kuendeleze ueledi wao wa kuunda visaidizi ili kuboresha uelewekaji wa dhana za Kiswahili. Kadhalika, washika dau wana changamoto kubwa ya kuleta mashuleni rasilimali ya kutosha kutengeneza visaidizi vinavyohitajika. Jambo lingine ni kuwa shule zingetenga sehemu mahususi ya kuhifadhi visaidizi ili vitumike bila uwoga wa kuharibika ama kupotea. Mwisho, walimu watie misingi thabiti ya ufunzaji msamiati kuanzia madarasa ya chini ili wanafunzi wasielewe dhana hizi kimakosa kisha wahitajike kurekebishwa baadaye. Jambo hili ndilo lililokita mizizi sehemu nyingi za Kaunti ya Kisumu Mashariki, Kenya.
\end{abstract}

Copy Right, IJAR, 2016,. All rights reserved.

\section{Utangulizi:-}

Kulingana na (Nunan: 2009), misingi ya ki-elimu ya kiasili, kisoshiolojia, kifisiolojia na kifalsafa, kumeibuka miondoko muhimu katika utendaji wa kufunza na kujifunza. Kufunza kuzuri ni kule kunakosababisha, kusuluhisha 
na kukuza ujifunzaji. Ili kufanikisha lengo hili, mwalimu, kama wafanyavyo mafundi wengine, sharti atumie mbinu almaarufu kama 'visaidizi vya kufunzia'. Visaidizi hivi ni maumbo mbalimbali yanayodhihirika mara kwa mara. Ingawa visaidizi vya kufunzia hutumika katika masomo, baadhi yazo hupotosha, huleta fikira potovu inayoletwa na kutokuwepo kwa makini fikira na matumizi kiwakati. Pendekezo hili la utafiti linawania kuchunguza jinsi matumizi ya visaidizi katika kufunza msamiati ili kuboresha uelewa wake katika shule za msingi, za umma wilaya ya Kisumu Mashariki, Kenya. Shabaha ya utafiti huu ni kudhihirisha sababu za kutotumia visaidizi hivyo kwa makini, kuchanganua na kutambua vikwazo vya matumizi ya visaidizi, kutoa ushahidi wa jinsi ambavyo visaidizi vya kufunzia vinavyofanikisha uelewa wa dhana zilizofunzwa.

Wasemavyo Just and Carpenter (2002), utafiti huu utatumia kiunzi cha kinadharia cha John Dewey kinachoitwa uasilia, vipengele vyake muhimu. Wazo hili pia linashikiliwa na Piaget (1945) huku akimnuku John Dewey kama mwanadhana shupavu katika mwanzo wa miaka ya 1900 .Alisema kwamba mchezo ni jambo fiche ambalo husaidia mtu pekee kukua kisoshiolojia na kiakili. Jambo hili lazima litengwe na kazi kwani mchezo husaidia mtoto kukua kama mtu mwenye ari ya kufanya kazi. Watoto wanapokuwa watu wazima huwacha mchezo na kutafuta motisha katika kazi zao. Jambo hili la kazi za mchezo humtayarisha kuwa mtu mzima aliye na afya na ari ya kazi. Uteuzi wa kusudi na sampuli utatumiwa kuchagua matumizi makinifu ya visaidizi vya kufunzia. Tomlinson (2012) anaeleza kuwa data ilikusanywa kwa njia ya mahojiano na kuchanganuliwa kwa kuzingatia ubora wake. Mtafiti alitumaini kuwa utafiti huu ungenufaisha shule za umma, Kisumu Mashariki, idara ya elimu, wanafunzi na walimu ili kudhibiti matumizi mabovu ya visaidizi, kutoeleweka kuzuri kwa msamiati na matokeo duni ya wanafunzi. Kujifunza ni matokeo ya mwingiliano kati ya mwalimu, wanafunzi, wazazi na jamii kwa jumla. Matokeo ya ujifunzaji hujitokeza katika mpangilio wa kujifunza unapo buniwa. Matokeo haya hutegemea pakubwa mpangilio uliopo wa shule katika ujifunzaji. Uelewekaji wa msamiati hutegemea visaidizi vya ujifunzaji vinavyo tumika kurahisisha somo (Brown, Oke and Brown: 2006).

Mjini Kisumu, mtihani wa kitaifa baada ya uchanganuzi data za shule kumi za umma, matokeo yamekuwa duni sana hasa kwenye somo la Kiswahili. Kwenye mtihani wa KCPE uliofanyika mwaka 2011, 47 katika shule 118 zilipata maki chini ya maki 250 kwa maki 500 ambapo ni asilimia arubaini (40\%) ya jumla ya shule zote na maki asilimia hamsini (50\%) ya nambari yote husika. Mwaka 2010, shule 48 hazikupata alama 250 na alama asilimia arubaini na moja $(41 \%)$ ya shule zote. Kilingana na afisi kuu ya washika dau elimu Kisumu (Kisumu Municipal Education Office Performance Report mwaka 2011) shule tano za chini nne zilipata maki ya wastani 175.29, 186.37, 190.28, 194.01 na 191.86. Utafiti huu una dhihirisha maki duni katika matokeo ya mtihani wa kitaifa katika shule za umma Manisipa ya Kisumu, Kutoka (2013, Januari 29), mwaka 2012, data ya matokeo ya shule za chini tano za majirani ya Kaunti za Magharibi, Kaunti ya Kisumu, na kaunti majirani zili pata maki 162.8, 176.8, 197.0, 190.2 na 174.8. Hii ni kwa sababu ya kutotumia mbinu mwafaka na visaidizi vya ufundishaji msamiati. Kutokana na data hii basi ni wazi kwamba kuna haja ya utafiti ambao utatoa na kueleza umuhimu wa visaidizi vya kufundishia. Utafiti huu unalenga kutambua umuhimu wa matumizi ya visaidizi vya kufunza msamiati ili kuboresha uelewekaji msamiati shule za umma za Kaunti ya Kisumu. Jedwali lifuatalo linaonyesha matokeo ya mitihani ya darasa la tatu ya miaka miwili kudhibitisha kwamba kuna pengo linalohitajika kuzibwa. Matokeo ya Kiswahili vile vile ya darasa la nane katika shule hizo hizo ni duni sana, hivyo basi hitaji ni wazi kuzibwa.

Linganisho la matokeo ya Kiswahili katika KCPE na Darasa la 3

\begin{tabular}{|c|c|c|c|c|c|}
\hline \multirow{2}{*}{ SHULE } & \multicolumn{2}{|c|}{2011} & \multicolumn{2}{|c|}{2012} & \multirow{2}{*}{ JUMLA } \\
\hline & KCPE & STD 3 & KCPE & STD 3 & \\
\hline $\mathrm{A}$ & 71.05 & 71.35 & 65.65 & 67.43 & 130 \\
\hline B & 54.09 & 58.80 & 55.70 & 57.18 & 172 \\
\hline $\mathrm{C}$ & 58.15 & 42.63 & 62.04 & 39.41 & 114 \\
\hline $\mathrm{D}$ & 57.67 & 69.3 & 56.59 & 68.38 & 85 \\
\hline $\mathrm{E}$ & 57.32 & 43.25 & 65.76 & 50.3 & 94 \\
\hline $\mathrm{F}$ & 53.46 & 59.61 & 53.61 & 54.54 & 24 \\
\hline $\mathrm{G}$ & 59.65 & 59.65 & 60.33 & 60.51 & 105 \\
\hline $\mathrm{H}$ & 60.39 & 44.30 & 52.26 & 31.47 & 76 \\
\hline I & 53.46 & 58.80 & 58.51 & 57.18 & 86 \\
\hline $\mathrm{J}$ & 39.67 & 57.5 & 44.86 & 61.80 & 39 \\
\hline
\end{tabular}

Kiini: Kazi ya mtafiti (2013) 


\section{Lengo la Utafiti:-}

Kutambua sera zilizopo za ukaguzi kudhibiti kwamba kila msamiati hufunzwa kwa utumizi wa video, chati na vinyago ili kusaidia uelewekaji wa msamiati.

\section{Yaliyoandikwa kuhusu mada:-}

Elimu ni jambo la muhimu na haki ya binadamu wote (Washington: 2011). Elimu ni ufunguo wa kudumisha maendeleo ya nchi yoyote ile. Ndio ufunguo kwa uchumi na Serikali ya Kenya imeonyesha umuhimu huu. Serikali mwakani 2003 ilianzisha mfumo wa elimu ya bure katika shule za msingi ili kuhakikisha kila mwanafunzi hanyimwi haki yake ya elimu. Visaidizi vya kujifunzia vimeundwa kuwakilisha hali halisi kuwezesha kufunzia wanafunzi kuelewa dhana zinazokusudiwa kueleweka.Kuwakilisha hali halisi kwa njia ya kutumia visaidizi au vyombo vya kufunzia na kuondoa utata, ili dhana hizo zieleweke kwa urahisi. Mwanafunzi popote alipo aweze kupata kujifunza. Mwalimu anapo mpa nafasi ya kuona, kubuni dhana inayo zungumziwa kwa uhalisia. Visaidizi vya kufunzia vina umuhimu na ni vyombo ambavyo husaidia kuleta motisha katika kazi ya mwalimu na mazingira ya kujifunzia. (Kathleen: 2012).

Kenya National Examinations Council (2005), inalalama kuhusu matokeo duni ya mtihani huu wa kitaifa (KCPE). Imetaja jinsi mahitaji ya silibasi hukumbanana na changamoto nyingi. Walimu huandaa somo bila visaidizi muhimu vya ufunzaji basi msamiati ikaelewekwa kwa njia finu mno. Baada ya walimu kuteua masomo katika silibasi wao huwa hawajitayarishi vyema. Mfano: kutengeneza mandalizi somo na kuteua visaidizi mwafaka vya somo. Hivyo changamoto huwa nyingi ndiposa utafiti huu umejikita kutafiti kuhusu utumiaji visaidizi vya kufundisha msamiati katika somo hili. Somo hili linahitaji kufunzwa kwa kuzua hisia, misisimuko wa kuwahusisha wanafunzi wote. Krashen (2003), anasema kwamba lugha ya Kiswahili ikifunzwa kwa kuwahusisha wanafunzi, basi wataibuka kuwa watenda kazi wenyewe na wabunifu. Visaidizi halisi huzua uchangamfu wa kujifunza lugha, huzua motisha na uelewekaji bora zaidi au msamiati hueleweka. Kulingana na Birgen (2005), visaidizi halisi huguswa, hushikwa basi msamiati hukumbukwa kwa urahisi kuliko kile kilichobuniwa ama kuzungumziwa tu.

PDSI (Plan, Do, See, Improve) ni njia ya kufunza ambayo imejikita kwa utafiti wa Penny (2000) ambayo imesisitiza haya ya wanafunzi kufanya mipango bora ya ufundishaji na mazoezi ambayo yanahusu kujionea wenzao na kuboresha mazoezi hata zaidi kwa ujifundishaji halisia kwa kutenda. Ni jambo la muhimu kujua kwamba hamna mwanafunzi ambaye ni mjinga na asiyeweza kujifunza, wengine huwa tu, wanachukua muda (Republic of Kenya: 2006b), PDSI sharti itimiziwe kabisa ili ihakikishe kwamba wanafunzi wote wanaweza kupata au kuelewa dhana zote walizo funzwa.

Kama mikakati ya kutimiza ya PDSI, kufunza lugha kwa mawasiliano (CLT) ilibuniwa, jambo la muhimu ni kuboresha utendaji kazi wa wanafunzi (Students' Communicative Competence) kwa kuendeleza umilisi wa mingiliano ya watu vile vile umilisi somo; na kujipima na changamoto za ufundishaji wa kitamaduni (traditional teaching approaches) (Becher: 2005) Kulingana nao umilisi wa lugha ushupavu ni ujuzi wa mzungumuzaji na jinsi anavyoweza zungumza lugha katika sherehe tofauti; kwa maneno mengine ni uwezo wa kuzungumza kwa usawa kijumla katika miingiliano ya utamaduni katika muktadha wa mawasiliano. CLT inaamini kwamba kuwapa wanafunzi nafsi ya hali halisia ya utumiaji wa visaidizi vya ufundishaji darasani ni njia moja mzuri ya kujiendeleza. Hata hivyo si kusema kwamba wanafunzi wahimizwe katika uzungumzaji na kijiendeleza na kusahau kutilia mkazo katika sarufi iundao lile ambalo wanazungumzia.

CLT haikubaliani na wazo kwamba kufunza lugha kama kueleza dhana na drili za sentensi kwani huku kutazua hali ya kuwa na uwezo wa "linguistic ability" na hawawezi kujielezea katika hali halisi katika maingiliano ya kila siku. Haya ni kweli kwani mzungumzaji lugha ya Kiswahili sharti ajue jinsi ya kutumia lugha katika mawasiliano halisi. PDSI inaweza fikiwa ikiwa upangaji, ufanyaji kazi, uonaji, na urekebishaji utafanywa Hivi ni kusema utendaji kazi utadhibitika na sio tu kuzungumza. (Wells: 2006) utendaji kazi huzua ubunifu, ari ya kufanya mazoezi mwafaka na kuwezesha wanafunzi kutumia lugha sahihi. Katika mpangilio wa Willis Model" wa utendaji kazi kazi kama anavyorejelea (Wringe: 2010) kihunzi hiki kimegawanywa katika hatua tatu.

Katika hatua ya kwanza, mwalimu anaeleza mada na anaweza kuwasaidia wanafunzi kukumbuka somo la awali litakalowasaidia katika utendaji kazi huo. Hii ni kama vile kutazama kanda za video za watu wakitenda kazi inayohusika ya kuonyesha ujumbe au visaidizi vingine. Hii huwezesha wanafunzi kubuni wanalotarajia, wanafunzi wanaweza kuchukua na kuandika daftari kazi fulani au kutumia mada kujitayarisha kwa kazi. Hatua ya pili ni kupanga kazi katika makundi au wawili wawili. Baada ya kujadili hoja, wao hutoa ripoti la kundi husika. Mwalimu huwa mwelekezi, msaidizi kisha wana kikundi huwasilisha kazi yao kwa mbinu ya orali au kusoma walio andika. Wanafunzi wengi husikiza na kukagua kazi ya makundi hayo. 
Mwalimu pamoja na wanafunzi wengine hukagua na kutoa zao. Hatua ya mwisho ni ile ya ukaguzi. Visaidizi vya kufunzia vimefanywa katika makundi mbalimbali. Kundi la visaidizi vilivyochapishwa, visaidizi halisi, visaidizi vya kutazama na mwisho visaidizi vya kusikiliza. Marine and Hilles (2008) anatambua kuwa visaidizi hivi ni vile vinavyoweza kuonekana na wanafunzi ambao huwasilisha ujumbe fulani kwa madhumuni ya kufunzia darasani. Aina hii ni kama picha, kadi za umeme, michoro, ramani, chati, visaidizi halisi. Ni dhahiri kwamba matumizi ya visadizi huchangia katika ufanisi wa kufundisha msamiati ya Kiswahili. Kyriacou (2001), anatoa kauli kwamba ni vyema kutumia viyango hivyo na kuvielewa kama anavyorejelea. Mwalimu anaweza eleza msamiati fulani kwa urahisi atumiapo visaidizi hivyo na uelewekaji msamiati ukafaulu. Visaidizi halisi huibua motisha na husaidia uelewekaji msamiati kwa urahisi mno. Wanafunzi hushika na huona basi kuweza kurudiarudia utendaji huo, hapo basi uelewekaji ukawa rahisi na kuweza kukumbukwa vyema. Visaidizi halisi vikilinganishwa na neno husika huwa rahisi kueleweka kuliko kubuni jambo katika ubongo (Just and Carpenter: 2002). Mwalimu huzungumza kwa uchache na mwanafunzi ndio mtenda kazi. Utendaji kazi unahimizwa katika mfumo mpya wa elimu. Wanafunzi wawe wavumbuzi wanapoingiliana na visaidizi katika somo.

\section{Mbinu Za Utafiti}

Kulingana na Kasomo (2007), sehemu hii ilishughulikia mpangilio wa utafiti, njia za utafiti na mbinu zilizotumiwa kukusanya data ili kutimiza madhumuni ya utafiti wetu. Kulikuwa na njia mseto zilizotumika kupata data ya uhakika. Utafiti ulifanywa maktabani na nyanjani. Nilisoma maswali yanayohusiana na mada hii mahususi. Data kuhusu swala la utafiti ulikusanywa nyanjani kupitia mahojiano na hojaji. Kwa kiwango kikubwa utafiti huu ulifanyika nyanjani na darasani. Mtafiti alijishushulisha zaidi na uchunguzi wa visaidizi vinavyotumiwa na walimu katika somo la msamiati katika darasa la tatu. Mtafiti alihudhuria somo na kukagua jinsi visaidizi vilivyoorodheshwa vilivyotumika.

Idadi Ya Wanafunzi wa Kiswahili

\begin{tabular}{|c|c|}
\hline Shule/Kiwango & Darasa la 3 \\
\hline A & 75 \\
\hline B & 80 \\
\hline C & 85 \\
\hline D & 90 \\
\hline E & 80 \\
\hline F & 75 \\
\hline G & 80 \\
\hline H & 85 \\
\hline I & 85 \\
\hline J & 90 \\
\hline JUMLA & $\mathbf{8 2 5}$ \\
\hline 10\% & $\mathbf{8 2}$ \\
\hline Kila Shule & 8 \\
\hline
\end{tabular}

Kiini: Kazi Ya Mtafiti (2013)

Idadi ya walenga ilikuwa walimu thelathini katika shule za msingi, za umma kama walivyoteuliwa katika maeleza haya.

Idadi Ya Walimu Wa Kiswahili

\begin{tabular}{|c|c|c|}
\hline SHULE/KIWANGO & WALENGWA & JUMLA \\
\hline A & 3 & 1 \\
\hline B & 2 & 1 \\
\hline C & 3 & 1 \\
\hline E & 4 & 1 \\
\hline F & 3 & 2 \\
\hline G & 2 & 1 \\
\hline I & 3 & 2 \\
\hline J & 4 & 1 \\
\hline JUMLA & 3 & 13 \\
\hline
\end{tabular}


Kutambua sera zilizopo za ukaguzi kudhibiti kwamba kila msamiati hufunzwa kwa utumizi wa video, chati na vinyago ili kusaidia uelewekaji wa msamiati.

Walimu Wakuu pamoja na Walimu wa Kiswahili waliulizwa iwapo shule zao zilikuwa na sera ya kudhibiti matumizi mwafaka ya visaidizi madarasani na matokeo ya majibu yao yalikuwa hivi:

Sera ya kudhibiti matumizi mwafaka ya visaidizi madarasani

\begin{tabular}{|c|c|c|c|c|}
\hline & \multicolumn{2}{|c|}{ Ndiyo } & \multicolumn{2}{c|}{ La } \\
\hline & f & $\%$ & F & $\%$ \\
\hline Msamiati wa wanyama & 3 & 37.5 & 5 & 62.5 \\
\hline Miti na mimea & 0 & 0.0 & 8 & 100.0 \\
\hline Msamiati wa vyakula & 5 & 62.5 & 3 & 37.5 \\
\hline Msamiati wa ndege & 0 & 0.0 & 4 & 50.0 \\
\hline
\end{tabular}

Katika Msamiati wa wanyama, $62.5 \%$ walichagua La nao $37.5 \%$ wakachagua Ndiyo. Katika miti na mimea, wote (100.0\%) walichagua La. Katika msamiati wa vyakula, $37.5 \%$ walichagua La nao $62.5 \%$ wakachagua Ndiyo. Katika msamiati wa ndege, $50.0 \%$ walichagua La nao $50.0 \%$ wakachagua Ndiyo. Walieleza sababu zao kuchagua majibu hayo ifwatavyo: Ni wazi kwamba sehemu fulani hazishughulikiwi na wakati mwingine zinahepwa. Silibasi inaeleza wazi jambo hili kwa hivyo litekelezwe. Mitihani ya kitaifa imeonyesha jambo hili wazi kwani wakitahini vipengele vya madarasa ya chini matokeo huwa duni hadi walimu wanatete jinsi mtihani ulivyo mgumu eti vipengele vile havimo silibasini. Huu ni mwamko mpya lazima walimu wajihami na silibasi wajue mahitaji ya kila darasa ni vipi watawasilisha ujumbe na ueleweke kwa urahisi. Silibasi imeorodhesha vifaa vyote vya kila darasa hivyo walimu wasipuzilishe hayo. Kuhusu sera iliyokuwa shuleni mwao ya kubuni visaidizi vya kufundisha sehemu teule za lugha ya Kiswahili, Walimu Wakuu pamoja na Walimu wa Kiswahili walijibu na wanafunzi hivi:

Msamiati wa vyakula hamna iwapo ni jukumu la mwalimu kufwata silibasi na kutengeza. Sera hasa ya shule kutengeza hamnalakini kila mwalimu anahimizwa kuunda vifaa hivyo.Changamoto vilevile zinazidi kutokana na wingi wa wanafunzi, sehemu za kuhifadhi, senti za kununua visaidizi.

Msamiati wa wanyama wapori hamna kujadili haya hata wanapo fikiria kuzuru sehemu mbalimbali huwa ni katika somo la geografia. Walimu hawafikirie kutumia wakati huo kuelimisha wanafunzi msamiati wa wanyama ingawa wengine pia wana shida ya utambuzi huo.

Msamiati wa ndege wa pori hamna wakati jopo hujadili haya. Muhimu ni kuhusu matokeo bora ndiyo kiini mgogo wa shule zote bila kukumbuka kwamba matokeo bora huanzia kwenye ufundishaji mwema.

Msamiati wa mimea na miti jopo hupata vikao lakini hamna sera za kuunda vifaa vya kufunzia. Wakati mwingi tuu ni mambo ya kutengeneza ratiba ya somo, maandalizi somo, kusahihisha vitabu na matokeo ya mitihani.

Walimu Wakuu pamoja na Walimu wa Kiswahili na wanafunzi walipoulizwa iwapo shule ilikuwa na sera ya kutoa ukaguzi wa machanganuzi ya visaidizi vya ufundishaji wa sehemu mbalimbali za lugha madarasani, 37.5\% walichagua Ndiyo nao 62.5\% wakachagua La na wakaeleza sababu za kuchagua majibu yao ifwatavyo:Jopo huwa halina mtindo huo pengine kuwe na wakanguzi kutoka idara ya elimu,walimu wakuu ambao hukangua walimu wa kiswahili. Walimu wengi walipoulizwa jambo hili kwa sababu hawapati vifaa hivi. Kuhusu kiwango cha mapato ya shule yaliyotumika kununulia visaidizi vya ufundishaji wa sehemu mbalimbali za lugha, Walimu Wakuu pamoja na Walimu wa Kiswahili waliashiria matokeo haya: $5.56 \%$ asilimia 5 au chini, $27.78 \%$ asilimia 5 hadi $16.67 \%$ asilimia 21 hadi $25,22.22 \%$ asilimia 26 hadi 30 na $27.78 \%$ asilimia 31 au zaidi.

Kuhusu swali iwapo walimu walipata warsha za kuboresha ubunifu wa matayarisho ya visaidizi vya ufundishaji wa sehemu mbalimbali za lugha, Walimu Wakuu pamoja na Walimu wa Kiswahili wote (100\%) walijibu La na kueleza sababu ya kuchagua majibu yao hivi:Kudhibitisha hayo washika dau elimu huzuru shule kadha tuu ambapo nia yao ni kuchunguza mwalimu anavyofunza. Wakati mwingi wao huangalia ratiba, maandalizi somo, usafi, idadi ya wanafunzi na matokeo ya mtihani wa kitaifa. Warsha vilevile huelekezwa kwenye mbinu za uandishi insha au vitabu ambavyo vinavyotumika kufunzia, vitabu vya kiada. Warsha za kuunda visaidizi au za kuboresha jopo katika kumbuni sera za kuunda vifaa hivi vya kufunza havijambobea. Ni jambo la kujadiliwa kwani kiswahili bado hakifanyi hivi.Ni vyema kila shule ibuni jopo la kuangalia matumizi ya visaidizi, utengenezaji na kuhakiki kwamba 
uzoefu wa kutumia visaidizi hivi umetia fora na kutumika wakati wote ili vikasaidia uelewa dhana.Kwani kutumia mara moja ni kama kutotumia kabisa.

Wahojaji walipoulizwa iwapo kulikuwa na mikakati ambayo iliyowekwa kuzingatia mahudhurio katika warsha za kuboresha ubunifu, $62.5 \%$ walijibu Ndiyo nao $37.5 \%$ wakajibu La. Walitoa sababu zifwatazo kuelezea sababu za kuchagua majibu yao. Walipoulizwa iwapo walimu wa somo la Kiswahili huwa na vikao vya kujadili machanganuzi ya visaidizi vya ufundishaji wa sehemu mbalimbali za lugha katika masomo, Walimu Wakuu pamoja na Walimu wa Kiswahili wote $(100 \%)$ walijibu La na na kueleza sababu ya kuchagua majibu yao hivi:Wakati uchanganuzi unafanyika huwa wa vipengele kama matokeo ya mitihani,utaratibu wa kufunza,ratiba,maandalizi somo. Sera ya kuchanganua utumizi wa visaidizi haupo katika Kaunti hii ya Kisumu. Walimu Wakuu, Walimu wa Kiswahili na wanafunzi wote $(100 \%)$ walijibu Ndiyo walipoulizwa iwapo walidhani kuwa matumizi ya visaidizi vya ufundishaji wa msamiati ya somo la Kiswahili uliadhiri matokeo ya mtihani. Walitoa sababu zifwatazo kuelezea sababu za kuchagua majibu yao:Msamiati hutatiza wanafunzi sana wanapoulizwa kujieleza kwa lugha hii.Wengi wao husema kwamba hawajui mambo fulani katika lugha ya kiswahili hivyo utata katika kutumia kwa mfululizo mzuri.Mfano wana funzi ambao hufanya vizuri katika mitihani yao ya kitaifa huo wanashinda ya kujieleza vizuri.Miingiliano ya mawasiliano huo shida kwa kiwango kikubwa tuu.Shida hii ime zua utata wa wahutubi.wasomaji habari,weledi katika ku jieleza vyema katika hadhara fulani kama ilivyo katika kiingereza.Kulingana na Su and Hong (2007), utumiaji wa visaidizi husaidia pakubwa mno.

\section{Mukhtasari, hitimisho na mapendekezo:-}

Katika sura hii, mtafiti anajadili matokeo ya utafiti kwa muhtasari, kutoa hitimisho na kufanya mapendekezo juu ya mambo yanayohusu matumizi bora ya visaidizi vya ufundishaji msamiati wa somo la kiswahili katika kaunti ya Kisumu mashariki, Kenya.

\section{Muhtasari:-}

Somo hili ni muhimu sana kwa taifa letu la Kenya ili kuboresha utamaduni wetu. Somo hili huwawezesha watu kila sehemu kuwasiliana bila utata na kuwafanya kuwa na mwingiliano mwafaka. Igawa changamoto ni nyingi katika kufundisha msamiati ni bora kutilia mkazo ili kuboresha uelewa wa somo hili kama mengine. Mielekeo hasi ya walimu na wanafunzi iondolewe kabisa.Visaidizi vinavyotakikana kutumiwa vitumiwe vilivyo kuliko kuepukana utumizi wa visaidizi hivi.

\section{Hitimisho:-}

Lengo la utafiti huu lilikuwa ni kuchunguza matumizi ya vifaa vya ufundishaji msamiati ya somo la Kiswahili, utafiti ulilenga kuboresha somo la Kiswahili. Miongoni mwa visaidizi vilivyotafitiwa, watafiti wengine wanaweza kutafiti visaidizi vingine katika vipengele vingine, kwenye upeo na mipaka mingine, kwa lengo la kudhibitisha kwamba visaidizi vina ufaafu au udhaifu katika kujifunza na kufundisha msamiati ya kiswahili. Utafiti ulinuia kukagua sera za kutengeneza visaidizi vya kufunzia msamiati.Utafiti huu una wahimiza watafiti wengine kushughulikia matumizi ya nyenzo katika vipengele vingine vya lugha madarasa ya kiwango cha juu kwani nililenga tuu darasa la tatu. Sehemu nyingine ya kutafitwa ni vipi sera za kuunda au kuhifadhi nyenzo hizi zaweza kuboreshwa ili zilete ufaafu zaidi katika elimu ya kiwango chochote kile.Warsha pia ni muhimu kuleta motisha kwa walimu wa lugha hii ili wanafunzi wabadili mielekeo hasi kuhusu somo hili, hivyo kuboeresha matokeo ya mitihani. Ili kufanikisha lengo hili, walimu wakuu, walimu wa somo hili, wanafunzi walipewa hojaji za kujaza ili waweze kujaza pengo zilizowachwa ili kupata uhakika uliopo katika matumizi ya vifaa katika somo hilo. Kutokana na hojaji na mahojiano haya tuliweza kupata data ambazo, baada ya uchanganuzi, zilitoa picha halisi ya vifaa hivyo.

Kutokana na mfumo wa elimu wa 8-4-4, inatokea kuwa mara nyingi, walimu, wazazi na hata wanafunzi wanapitia changamoto nyingi katika shughuli za masomo hasa wanapolenga kupata maki za juu katika mitihani ya kitaifa ilhali kule kutia misingi bora katika dhana za msamiati hatiliwi maanani. Huku huzusha waliopita na waliolemewa na lugha, hasa wanaopohitajika kujiendeleza maishani wakitumia lugha Taifa hii hii. Kwa mfano wanafunzi huzuka na alama ya A katika mitihani ya Kitaifa lakini huwa katika miingiliano ya mawasiliano hawajiwezi vilivyo. Hii ni hali halisia nchini Kenya inaleta taharuki, na ndiyo iliyonipa motisha kutafiti mada hii ili niweze kulimulika jambo hili kwa makusudi ya kuwazuzua wasomi, wataalamu na watafiti wengine kuendeleza kuchambua tatizo sugu hili. 


\section{Marejeleo:-}

1. Allinder, R.M. (2001). Improving Fluency in At-Risk Readers and Students with Learning Disabilities.Remedial and Special Education 22, 1: 48-55.

2. Becher, R.M. (2005). Parent Involvement and Reading Achievement: A Review of Research and Implications for Practice. Childhood Education, 62 (1), Sept/Oct, 42-50.

3. Birgen, P. (2005). A Teacher can Break or Make a Child.Education Insight. Nairobi: Insight Publishers.

4. Brown, R.N., Oke, F.E., and Brown, D.P. (2006).Curriculum and Instruction: An Introduction to Methods of Teaching. London: Macmillan Publishers.

5. Cohen, L. \& Manion, I (1994). Research methods in education. London: Rutledge

6. Chall, J.S., Jacobs, V.A. and Baldwin, L.E. (2007).The Reading Crisis: Why Poor Children Fall Behind. Cambridge, Mass: Harvard University Press.

7. Creswell, J.W. (2003). Research Design: Qualitative and Mixed Methods Approaches. $2^{\text {nd }}$ Ed. London: Sage Publication.

8. Dewey, J. (2009). John Dewey between Pragmatism and Constructivism.Fordham American Philosophy. Fordham University Press,

9. Flavell, J. H. and Piaget, J. (1963) Developmental Psychology of Jean Piaget, Princeton,

10. Foorman, R. R. and Torgesen, J. (2001). Critical elements of classroom and small-group instruction promote reading success in all children. Learning Disabilities Research and Practice, 16(4):203-212.

11. Gelzheizer, L. L. and Meyers, I. (2008).Reading Instruction by Classroom, Remedial, and Resource Room Teachers.Journal of Special Education, 24(4), 512-527.

12. Goffin, S.G., Wilson C. (2001). Curriculum Models and Early Childhood Education: Appraising the Relationship ( $2^{\text {nd }}$ Ed). New Jersey: Merrill/Prentice Hall.

13. Grove, M.C. and Hauptfleisch, H.M.A.M. (2008).Remedial Education in the Primary School.Pretoria: HAUM Educational Publishers.

14. Just, M.A. and Carpenter, P.A. (2002).The Psychology of Reading and Language Comprehension.Boston: Allyn and Bacon.

15. Kahmi, A.G. and Catts, H.W. (2006).Reading Disabilities: A developmental languageperspective. Boston: College-Hill.

16. Kasomo, D. (2007). Research Methods in Humanities and Education. $\left(2^{\text {nd }}\right.$ Ed). Nairobi: Kijabe Printing Press.

17. Kathleen, A.M. (2012). Developing a Questionnaire for use in Outcomes Assessment. Buckwell University: Institute of Research and Assessment.

18. Kenya National Examination Council (2005).Kenya Certificate of Secondary Education 2004 Candidates Performance Report. Nairobi: Kenya National Examination Council.

19. Krashen, S. (2003).The Power of Reading.Englewood, Colorado: Libraries Unlimited.

20. Kyriacou, C. (2001). Essential Teaching Skills ( $2^{\text {nd }}$ Ed). Delta Place 27 Bath Road Cheltenham U.K: Nelson Thomas Ltd.

21. Marine C.M., Hilles S. (2008).Techniques and Resources in Teaching Grammar.Oxford University Press.

22. Mogeni, J.M. (2005). Factors Influencing Utilization of Resources in Teaching Kiswahili in Selected Public Secondary Schools in Trasmara District, Kenya.MA Thesis. Nairobi: Kenyatta University.

23. Mugenda, O.M. \&Mugenda, A.G. (2003).Research Methods: Qualitative and Quantitative Approaches. Nairobi: African Centre for Technology Studies Press.

24. Njogu, K. \& Nganje.D.K. (2006) Kiswahili Kwa Vyuo Vya Ualimu, Nairobi; jomo Kenyatta Foundation.

25. Nunan, D. (2009). Language Teaching Methodology: A Text Book for Teachers. London: Prentice Hall International.

26. Orasanu, J. (2001). Reading Comprehension.From Research to Practice.Hillsdale: Lawrence Erlbaum.

27. Orodho, J.A. (2003) Regional Inequalities in Education, Population and Poverty Patterns in Kenya: Emerging Issues and Policy Directions, Population of Kenya Journal,

28. Orodho, J.A. (2005). Elements of Education and Social Sciences Research Methods. Nairobi: Masola Publishers.

29. Penny, U.R. (2000). A Course in Language Teaching: Practice and theory. Cambridge: Cambridge University Press, ISBN-0521-44994-4.

30. Piaget, J. (1945). The Psychology of Intelligence. New York: Routledge Publications.

31. Pretorius, E.J. (2000). Reading and the UNISA Student: Is Academic Performance Related to Reading Ability. Progression, 22(2): 35-48. 
32. Pretorius, E.J. (2002). Reading Ability and Academic Performance in South Africa: Are we fiddling while Rome is burning? Language Matters, Issue 33, 169-196.

33. Quist, D. (2005). Primary Teaching Methods. London: Macmillan Publishers.

34. Republic of Kenya (1964) Kenya Education Commission Report. Nairobi: Government Printer

35. Republic of Kenya (2006a).Early Childhood Development Service Standard Guidelines for Kenya. Nairobi: Government Printer.

36. Republic of Kenya (2006b).National Early Childhood Development Policy Framework. Nairobi: Government Printer.

37. Riley, J.L. (2005). Learning in the Early Years (A Guide for Teachers of Children 3-7). London: Paul Chapman Publishing Ltd.

38. Roux, L.E. (2001) the quality of pupil participation in ESL classroom: an evaluation Unpublished MA Dissertation. University of Portcheftsroom.

39. Su, M.J. \& Hong, Z.F. (2007).Professional development of science practice teachers: Application of teaching aids. London: Paul Chapman Publishing Ltd.

40. Tomlinson, B. (2012). Materials Development in Language Learning. Cambridge: Cambridge University Press.

41. Vivas, E. (2011).Effects of Story Reading on Language.Language Learning, 46: 189-216.

42. Wang, M.C. (2009). Design and Manufacturing of Teaching Aids for Early Children. Taipei: Farterng.

43. Washington, J.A. (2011). Early Literacy Skills in African-American Children: Research Considerations. Learning Disabilities Research and Practice.16(4): 213-221.

44. Wells, G. (2006). The Meaning Makers: Children Learning Language and using Language to Learn. London: Hodder and Stoughton.

45. Wolfenson, O. (2000). The Role of Parents in Students Academic Performance in Bondo Districts. Unpublished MEd Thesis. Egerton University, Kenya.

46. Wringe, C. (2010). The Effective Teaching of Modern Languages. UK: Longman Ltd 